\title{
Morphology and changes of elemental surface composition of tungsten bombarded with carbon ions
}

Ivan Bizyukov

Kharkiv National University, Faculty of Physics and Technologies, 31 Kurchatov Ave., Kharkiv 61108, Ukraine

Andreas Mutzke, Ralf Schneider

Max-Planck-Institut für Plasmaphysik, EURATOM Association, Wendelsteinstr. 1, 17491

Greifswald, Germany

Alexander M. Gigler

Ludwig-Maximilians-Universität München, Sektion Kristallographie, Theresienstr. 41/II, D80333 München, Germany

Karl Krieger

Max-Planck-Institut für Plasmaphysik, EURATOM Association, Boltzmannstr. 2, 85748 Garching, Germany 


\begin{abstract}
Surface morphology has a strong influence on sputtering and implantation. A newly developed Monte-Carlo code SDTrimSP-2D simulates ion bombardment of surfaces with a 2D surface morphology (1D is depth and another dimension is lateral) defined by a vertical crosssection of the solid. The simulations allow to study numerically the interdependency of surface geometry and sputtering and implantation processes. Experimental results of the bombardment of W layers deposited on polished pyrolitic graphite with $6 \mathrm{keV} \mathrm{C}$ ions were used for comparison with results of the simulation. Free parameters of the code, particularly the so-called anisotropy coefficient, are calibrated by comparison of the macroscopic evolution of the elemental surface composition to the experimental results. After calibration, the code reproduces qualitatively the evolution of the shape of nano-scale surface structures observed by atomic force microscopy and by scanning electron microscopy. The histograms of the surface heights obtained by measurements and by the simulation show qualitative agreement. Local values of the $\mathrm{W}$ sputtering yield and $\mathrm{C}$ areal density, which are accessible only from the simulations, have been found to be strongly dependent on the nano-scale geometry.
\end{abstract}

Keywords: surface morphology, local ion-surface interactions, carbon implantation, tungsten sputtering, SDTrimSP-2D.

PACs numbers: $61.80 . J h, 68.03 . \mathrm{Hj}, 68.37 . \mathrm{Hk}, 68.37 . \mathrm{Ps}, 68.47 . \mathrm{De}, 68.49 . \mathrm{Sf}, 79.20 . \mathrm{Rf}$ 


\section{Introduction}

The application of three different elements as plasma facing materials is foreseen in the design of the International Tokamak Experimental Reactor (ITER) [1, 2, 3]. The divertor baffle region and probably the first wall will utilize tungsten coated tiles. Carbon based materials are planned to be used for the divertor target plates, which will be exposed to the largest heat and particle fluxes. Intensive sputtering of $\mathrm{C}$ leads to penetration of $\mathrm{C}$ atoms into the edge plasma, where they are ionized. $\mathrm{C}$ ions are accelerated by the sheath potential towards the in-vessel surface up to energies of several $\mathrm{keV}$ [4]. Erosion of tungsten is dominated by impact of impurity [4] ions with $\mathrm{C}$ expected to be the most common species incident in the divertor region. While sputtering by gaseous ions is already well understood, the bombardment of a W surface with ions of solid state elements shows a complicated behaviour with a competition between erosion of the surface due to sputtering and growth of a layer due to implantation of the projectile ions.

Results of first laboratory controlled experiments and simulations on bombardment of $\mathrm{W}$ surface with $\mathrm{C}$ ions were discussed in [5]. The authors had achieved good qualitative agreement between experimental data and results of TRIDYN simulations. However, they already mentioned that experimental results might be influenced by surface roughness and cleanliness. In a subsequent study [6] the differences between $\mathrm{C}$ and $\mathrm{CH}_{3}$ ion bombardment was investigated using the same setup as in [5]. In case of $\mathrm{CH}_{3}$ ions, simulations did not agree well with experimental data. This was explained by results of ex-situ Rutherford back-scattering analysis of the edge area of the bombarded spot, which revealed a non-uniform distribution of the ion flux over the surface not taken into account by the simulation.

The influence of surface roughness and surface cleanliness was quantified and minimized, and the effect of non-uniform bombardment was eliminated by replacing in-situ weight-loss measurements with in-situ ion beam analysis [7]. These improvements have allowed to achieve good quantitative agreement between experimental results and simulations in case of smooth surfaces. It could also be demonstrated that surface roughness not only increases sputtering of tungsten, but also decreases implantation of carbon. Since surface roughness affects both processes, its presence may lead to incorrect interpretation of the results if compared to standard simulation codes like TRIDYN [8]. These are based on a binary collision model and assume perfectly smooth surfaces with the 1-D elemental modification of the solid calculated only as function of depth. To improve the simulations for situations where surface roughness can not be neglected, it is necessary to extend the existing binary collision Monte-Carlo codes.

Simulations were performed with the recently developed code SDTrimSP-2D, which is a 2-D mesh extension of SDTrimSP [9]. In turn, SDTrimSP is a generalized version of the TRIDYN program to be run in static or dynamic mode (SD) on sequential or parallel systems (SP). In contrast to existing binary collision codes including surface roughness in form of a fractal representation [10], SDTrimSP-2D simulates ion bombardment utilizing a 2-D mesh. The first dimension is the direction perpendicular to the macroscopic surface plane, the second one is one direction parallel to that plane.

This work discusses previously performed experiments [7] comparing the experimental data and SDTrimSP-2D simulations. In addition to data presented in [7], new data of the respective experiments obtained by atomic force microscopy became available. Comparison to experimental rersults has shown that the code is able to qualitatively reproduce modifications of the nano-scale surface morphology. The comparison is still of qualitative character because one needs to apply simplifications comparing 2-D modeling to the actual 3-D real surface morphology. Simulations also have shown the impact of the surface morphology on local parameters of ion-surface interactions, to which there is no direct experimental access. 


\section{Experimental techniques}

W surfaces were bombarded by mass-separated $12 \mathrm{keV} C_{2}^{-}$ions, resulting in each incident $\mathrm{C}$ projectile atom having energy of $6 \mathrm{keV}$ at normal incidence. As target magnetron-deposited $\mathrm{W}$ layers were used with surface roughness given by the roughness of the substrate. The mean projectile range of $6 \mathrm{keV} \mathrm{C}$ ions in pure $\mathrm{W}$ is $\mathrm{R}_{\mathrm{C}} \approx 10 \mathrm{~nm}$, while the average roughness value of the surface is $\mathrm{R}_{\mathrm{a}} \approx 200-300 \mathrm{~nm}$ depending on the size of the area chosen for averaging. Since $R_{a}>>R_{C}$, the surface roughness can not be ignored with respect to the modification of surface morphology and elemental composition of the surface.

Ion beam analysis (IBA) with $2.5 \mathrm{MeV}{ }^{3} \mathrm{He}$ ions was performed in-situ, monitoring the evolution of the surface composition with increasing fluence. The details of the experimental setup, as well as of the measurement procedures are described in [11], while the experimental results are reported in [7]. Ex-situ analysis of the sample surface was performed by secondary electron microscopy (SEM) and atomic force microscopy (AFM) after retrieving the samples from the vacuum target chamber.

Surface topography has been determined by means of a commercial Atomic Force Microscope (AFM) setup (Dimension 3100, Veeco, Santa Barbara, CA). Rectangular silicon cantilevers have been used in a tapping mode at approximately $80 \%$ of their free oscillation amplitude. The samples have been dusted off by a stream of dry nitrogen before imaging in order to avoid artifacts. The images have been obtained with a resolution of $512 \times 512$ pixels and $5 \times 5 \mu \mathrm{m}$. Processing, 3D-representation and analysis of the AFM data were done using the SPIP 4.3 (Image Metrology A/S, Lyngby, Denmark) software. Finally, data have been exported to an ASCII file-format to allow for a later comparison with SDTrimSP-2D.

Compared to SEM imagery, AFM has the advantage of measuring the surface topography on an absolute and calibrated scale. SEM also has a certain susceptibility to imaging artifacts due to edge effects at corners as well as difficulties by the different skin depths of different materials. Therefore, SEM images do not directly reveal height information like AFM. However, SEM has the advantage of a higher lateral resolution and of faster imaging compared to AFM. Consequently SEM was used to estimate the lateral uniformity of surface roughness features to ensure that detailed AFM analysis would not yield results distorted by singular artifacts.

\section{SDTrimSP-2D}

The binary collision code SDTrimSP follows projectiles (incident atoms) and target recoil atoms in full three-dimensional geometry until their energy falls below a preset value or until they leave the target. In SDTrimSP, only a target structure with one dimension (depth) is used to follow the dynamical change of the elemental composition as function of depth (and fluence of projectiles). In SDTrimSP-2D, a 2-D domain with separate cells is introduced (see Figure 1). In this case the surface morphology is included in one dimension with the second dimension again being the depth. In the second lateral dimension the surface is assumed to be uniform. In addition the code follows the density changes due to projectile and recoil particles coming to rest after complete slow-down at the end of their trajectories. In SDTrimSP, this is done by a 1-D relaxation of the cells. Volume changes of the cells are used to represent density changes keeping the volume density constant according to the material.

In SDTrimSP-2D, this procedure has been extended, such that all volume changes applied are divergence free in order to avoid artificial source terms. This reflects particle conservation in the projectile-target system expressed by volume changes. For each cell, these resulting mass fluxes are in addition modified by introducing the anisotropy coefficient $\left(K_{\text {anis }}\right)$ of the volume relaxation. This anisotropy coefficient defines the ratio of horizontal volume changes (representing horizontal mass fluxes parallel to the surface) and perpendicular volume changes (representing mass fluxes in vertical direction) as shown in Fig. 1. The horizontal transport 
(parallel to the surface) is usually set smaller than the vertical one, because experimentally most swelling and shrinking is observed in the vertical dimension. Additionally, splitting and annihilation of cells was introduced in SDTrimSP-2D according to maximum and minimum densities. Details of these newly implemented parts of the code will be described in [12].

It should be noted that the anisotropy coefficient is an unknown parameter due to resolving a lateral dimension in SDTrimSP-2D. In contrast to all other physics parameters, which are naturally inherited from the original TRIDYN code, there is no independent experimentally proved foundation for the value of the anisotropy coefficient. It may depend for example on surface composition, material stress, and temperature. In this work it is considered to be an initially free parameter, which is is varied to fit the results of the simulation to the experimental data. One has to take into account that the experimental data correspond to a 3D system, while the model includes only lateral variations in one dimension. Because of this approximation, the code allows only qualitative comparisons. However, comparison of simulations and experiment allows to estimate the anisotropy coefficient for a given target structure, which can then in turn be used for further studies of this particular system.

\section{Comparison between the experiment and the modeling}

A typical AFM image of the initial surface morphology is shown in Figure 2(a). The simulation is performed using a 2-D surface profile cut from the original AFM picture along the line denoted by the arrow. The cross-section of the 2-D cut is shown in Figure 3(a), where the edge parts of the simulation were omitted, because the edge of the incoming beam in the simulation produces numerical artifacts like cratering. The 2-D cut was chosen to provide both a pronounced morphology region and a relatively smooth part of the surface. The simulation was done up to a fluence of $3 \times 10^{18} \mathrm{~cm}^{-2}$, when the surface had been almost completely covered by the ion beam deposited $\mathrm{C}$ layer, protecting the virgin $\mathrm{W}$ surface from further sputtering (see Figure 3(d)).

\subsection{Evolution of elemental surface composition}

During the bombardment of the $\mathrm{W}$ layer with $\mathrm{C}$ ions the concentration of these elements in the layer is changed due to the $\mathrm{W}$ sputtering and the simultaneous implantation of $\mathrm{C}$. The local parameters of sputtering and implantation depend on morphology details and are not available by currently existing diagnostics. However, the change in areal density of both elements averaged over the analyzed area of $\varnothing 1 \mathrm{~mm}$ can be measured by ion beam methods and used for calibrating the value of the anisotropy coefficient $K_{\text {anis }}$ in the simulation. Figure 4 shows the evolution of the elemental surface composition as obtained from experiments and in comparison to the results of SDTrimSP and SDTrimSP-2D simulations with different $K_{a n i s}$ values. One can see that SDTrimSP simulations using just a 1-D grid of the depth below the surface have failed to predict even qualitative estimates because the evolution of the elemental surface composition is affected by surface roughness.

On the other hand, SDTrimSP-2D is able to fit experimental data varying $K_{\text {anis }}$ value. It can be seen that the sputtering of the $\mathrm{W}$ atoms is less sensitive to changes of the anisotropy coefficient than the implantation of $\mathrm{C}$. The $\mathrm{W}$ sputtering decreases with increasing $\mathrm{C}$ concentration in the mixed W-C near-surface layer until complete coverage with $\mathrm{C}$ will stop it. Formation of W-C layer proceeds approximately similar in the range of $K_{\text {anis }}=0.1-0.5$. The roughness of $\mathrm{C}$ layer itself (and consequently its sputter yield) strongly depends on the anisotropy coefficient (see Figure 5). The best match of the experimental results and the simulation was obtained using $K_{\text {anis }}=0.2$ (see Figure 4).

The impact of the value of the anisotropy coefficient on the morphology is shown in Figure 5. For $K_{a n i s}=0.0$, the surface morphology exhibits unrealistically sharp peaks, which disappear gradually when the anisotropy coefficient increases. The surface shape at $K_{a n i s}=0.5$ is too 
smooth, while at $K_{\text {anis }}=0.2$ the surface keeps a reasonable roughness level matching the experimental results.

\subsection{Ion beam modification of the morphology}

SEM images of the bombarded surface depicted in Figure 6 give a qualitative picture of the evolution of the $\mathrm{W}$ surface morphology under bombardment with $\mathrm{C}$ ions. The initial surface structure is characterized by a relatively flat surface studded with semi-spherical elements (Figure 6(a)). According to the AFM measurements (Figure 2(a)), the height of these protrusions is up to $200 \mathrm{~nm}$. After certain bombardment fluence $\left(\approx 10^{18} \mathrm{~cm}^{-2}\right)$ the semi-spherical shape of these elements is modified towards a more pointed shape as seen in Figure 6(b). This image was taken after completion of the bombardment experiment, however, located at the edge of the bombarded area, where the local ion beam fluence is significantly smaller than in the central part. This means, however, that the local fluence could be estimated only qualitatively. The increase of the fluence leads to the so-called planarization effect due to both further degradation of the initially semi-spherical elements and to thickening of the protective $\mathrm{C}$ layer (Figure 6(c)). However, a few peaks, which have not been completely eroded, still can be found sticking out from below the $\mathrm{C}$ layer. These peaks have not been observed by AFM, which shows only the morphology of the grown $C$ layer due to the smaller lateral resolution (Figure 2(b)).

The modification of the experimental surface 2-D profile under $\mathrm{C}$ ion bombardment was simulated with the SDTrimSP-2D code. The calculated evolution of the surface profile with increasing fluence is shown in Figure 3, where again only the central part of the 2-D cut marked by the line with arrows in Figure 2(a) is shown excluding the edge regions of the simulation. The numerical simulation has been performed with $K_{a n i s}=0.2$ and the input surface profile is depicted in Figure 3(a). Figure 3(b) and (c) show that calculations well reproduce the tendency to steepen the semi-spherical elements observed by SEM (Figure 6(b)). Disappearance of the roughness is due to the growth of the $\mathrm{C}$ layer, since the highest point of the considered peak is remaining at approximately $\approx 350 \mathrm{~nm}$ until the fluence exceeds $2 \times 10^{18} \mathrm{~cm}^{-2}$. The peak regions have lost the largest amount of $\mathrm{W}$, while the rest of the area has been covered by a $\mathrm{C}$ layer already after a fluence of $2 \times 10^{18} \mathrm{~cm}^{-2}$.

The simulation was followed until nearly all $\mathrm{W}$ was covered by a $\mathrm{C}$ layer (Figure 3(d)). The obtained surface profile can be compared to the experimentally observed profiles shown in Figure 2(b) and Figure 6(c). Most likely, the difference between the experimental and simulation fluence required for coverage by $\mathrm{C}$ film can be explained by the size of the chosen element, which may include features deviating from the experimental average. One can see that the surface in the simulation does not become perfectly smooth in the simulated fluence range and that the roughness still exists due to the remains of the semi-spherical surface element initially present in the 2-D cut (Figure 3). The remaining roughness after the coverage of the initial surface by a $\mathrm{C}$ layer is due to the volume relaxation in the simulation, explained in section 3 .

Surface roughness can be determined both experimentally and by simulation and compared using a histogram based analysis; however, one has to note that the statistics for the simulation is rather limited (Figure 7) due to the small length of the computational surface. The comparison of the histograms in Figure 7(a) along the 2-D cut (simulation) and over the 3-D cutted area (experiment, hashed rectangle in Figure 2(a)) shows good qualitative agreement, which demonstrates that both representations of the surface morphology agree within the statistical error. One should take into account, that absolute height (i.e. position of histograms on abscissa axis) was adjusted to fit histograms each other. The 3-D cutted area was chosen to include the area around the semi-spherical element, which is present in the 2-D cut. Figure 2(b) shows the surface after the bombardment with the 3 -D cutted area chosen to represent a region of small height scattering, but typical for the entire observed area.

Comparison of the histograms after the bombardment shows good qualitative agreement between simulation and experiment reflecting the narrowing of the initially broad height 
distribution by the smoothening effect (Figure 7(b)). It also confirms the estimation of the chosen anisotropy coefficient, since it affects the medium range surface morphology and, thus, the resulting height distribution. The bimodal distribution, achieved in simulation, is conditioned by two regions at two different levels (see Figure 3(d)): C layer surface at $300 \mathrm{~nm}$ height and area around the small $\mathrm{W}$ peak at $220 \mathrm{~nm}$ height. The difference between these two levels corresponds to difference between the peaks of simulated histogram in Figure 7(b). One assumes that increasing statistical quality of simulation would smooth out these two peaks.

\section{Local characteristics of ion-surface interactions}

One of the most interesting tools of the code is the local diagnostics of ion-surface interactions, like sputtering yields, areal densities, etc, which are not accessible by conventional ion beam analysis methods. This tool, however, reveals the dependencies of these parameters on geometrical properties of the sample morphology. For the discussed bombardment of tungsten by carbon, the most interesting parameters are the sputtering yield of $\mathrm{W}$ and the areal density and depth distribution of implanted $\mathrm{C}$ atoms. The sputtering yield of $\mathrm{W}$ atoms was calculated by the code as the difference in $\mathrm{W}$ areal density between two subsequent fluence steps. Figure 8 shows the distribution of these parameters along the profile of the surface and their change with fluence. Comparing Figure 8 with Figure 3 one can clearly separate two principally different regions: one with a relatively smooth surface profile and another one containing the semispherical protrusion.

The relatively smooth surface is characterized by a faster growth of a $\mathrm{C}$ layer and one observes that already at a fluence of $1 \times 10^{18} \mathrm{~cm}^{-2}$ the sputtering yield of $\mathrm{W}$ drops by one order of magnitude. In contrast, the area with the semi-spherical protrusion is sputtered at a higher rate. After a fluence of $1 \times 10^{18} \mathrm{~cm}^{-2}$, this is the only area, where $\mathrm{W}$ sputtering still provides a significant contribution to the macroscopic decrease of the $\mathrm{W}$ areal density observed in the experiment. The highest sputtering yield is observed at the slope of the peak structure, due to the higher local incidence angle of the ions. Sputtering of $\mathrm{W}$ continues in the peak region even at and above a fluence of $3 \times 10^{18} \mathrm{~cm}^{-2}$, while the 1D standard version of SDTrimSP without surface morphology predicts pure carbon coverage at the surface after a fluence of $2 \times 10^{18} \mathrm{~cm}^{-2}$.

From the simulation one can conclude that the faster decrease of the $\mathrm{W}$ areal density and the slower growth of a $\mathrm{C}$ layer with increasing fluence is mainly due to the effect of the semispherical protrusions at the sample surface. Even if only small fractions of the surface are composed of such structures, (as for the sample in Figure 6, (a) and (b)) they have a significant effect on the experimental results due to the one order of magnitude higher sputtering yield at elevated local incidence angle.

\section{Conclusions}

In simulations of carbon bombardment of rough tungsten surfaces, nano-scale surface structures have been found to significantly affect sputtering of surfaces and implantation of projectiles. Binary collision simulations using just a 1-D grid of the depth below the surface have failed to predict even qualitative estimates. A new code, SDTrimSP-2D, has been developed to provide a more realistic model of the effects of surface morphology on sputtering and implantation processes. The code allows monitoring of the dynamic modification of the surface morphology under ion bombardment and provides the tools for studying local parameters of ionsurface interactions. This allows to understand the macroscopic results as a consequence of surface geometry on the nano scale and its impact on local parameters to which there is no direct experimental access. The anisotropy coefficient was a free parameter in the simulation and has been used to match the simulation with the experimental data on $\mathrm{W}$ sputtering and $\mathrm{C}$ implantation. However, one should note that the simulation represents a $2 \mathrm{D}$ system with one 
lateral dimension still assumed uniform, while the real surface is generally varying in all spatial dimensions. Therefore, only qualitative conclusions can be drawn from comparison of the simulations with experimental data.

By adjusting the anisotropy coefficient, it is indeed possible to reproduce the experimentally observed evolution of the surface morphology by the $2 \mathrm{D}$ simulation with the aforementioned caveat. Particularly the evolution of the calculated height distribution of the 2-D surface profile agrees qualitatively with the corresponding evolution of the distribution of the topographic AFM image. The simulation shows that the local angle of incidence resulting from a given surface morphology is the most relevant quantity determining $\mathrm{W}$ sputtering and $\mathrm{C}$ implantation rates. Surfaces with strong height gradients were shown to be sputtered most effectively, while the implantation rate of $\mathrm{C}$ in the same area remains approximately constant until the protruding parts of the nano-scale surface structure are almost completely sputtered and covered by a closed $\mathrm{C}$ layer. In contrast, relatively smooth parts of the surface are covered much faster with a closed $\mathrm{C}$ layer. On average, however, nano-scale roughness slows down the $\mathrm{C}$ implantation and increases $\mathrm{W}$ sputtering rates, which corresponds to the experimental findings .

\section{Acknowledgements}

Ralf Schneider acknowledges funding of the work by the Initiative and Networking Fund of the Helmholtz Association. The authors would also like to thank H. Lee for valuable technical assistance.

\section{References}

[1] G. Janeschitz, ITER JCT and ITER HTs, J. Nucl. Mat. vol. 290-293 p.1 (2001).

[2] D. Meade, et al., Mission and design of the fusion ignition research experiment, in: Proceedings of the 18th IAEA Conf. on Fusion Energy, Sorrento, Italy October, 2000, (CD-ROM), pp. IAEA-CN-77/FTP2/16, IAEA, Vienna, 2001.

[3] S. Nishio, et al., Conceptional design of advanced steady-state tokamak reactor, in: Proceedings of the 18th Conf. on Fusion Energy, Sorrento, Italy October, 2000, (CDROM), pp. IAEA-CN-77/FTP2/14, IAEA, Vienna, 2001.

[4] K. Krieger et al., J. Nucl. Mat. Vol.266 -269 p.207 (1999).

[5] W.Eckstein, J.Roth, Nucl. Instrum. and Methods in Phys. Res., vol.B53, p.279 (1991).

[6] W. Eckstein, K. Krieger, J. Roth, J. Nucl. Mat. Vol.258-263 p.912-916 (1998).

[7] I. Bizyukov, K. Krieger, N. Azarenkov, U. von Toussaint, J. Appl. Phys. Vol.100, p.1, 
Page 9

(2006).

[8] W. Moeller, W. Eckstein, J.P. Biersack, Comput. Phys. Commun. Vol.51 p.355 (1988).

[9] W. Eckstein, R. Dohmen, A. Mutzke, R. Schneider. SDTrimSP: A Monte-Carlo Code for Calculating Collision Phenomena in Randomized Targets, IPP 12/3, 2007

[10] N. Rutzic, Nucl. Instrum. Methods B47 issue 2 (1989) p. 118

[11] I. Bizyukov, K. Krieger. Rev. Sci. Instrum. 77 (2006) 043501

[12] A. Mutzke, R. Schneider. to be published 


\section{List of figures}

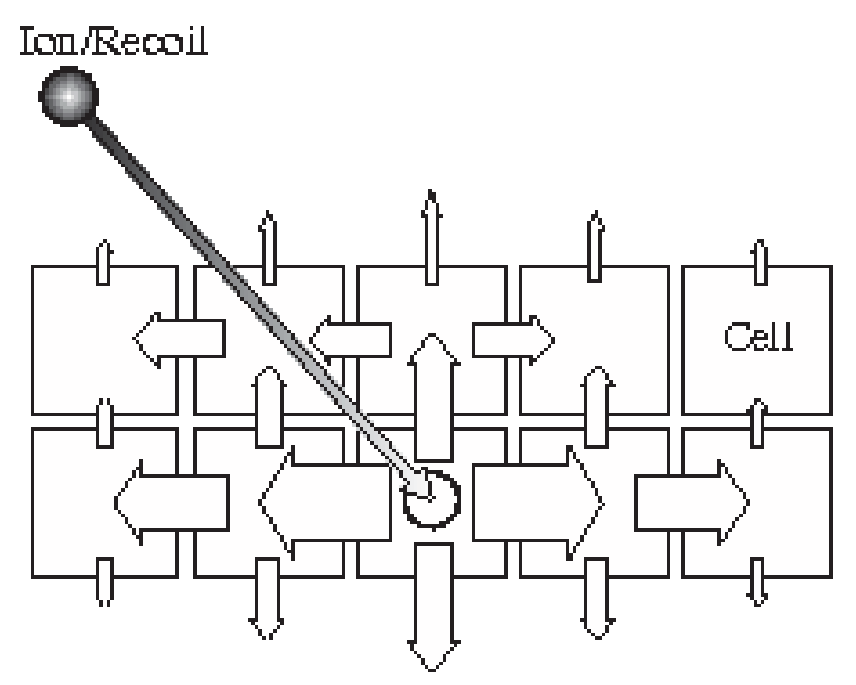

Figure 1. Schematic view of the 2-D cut into the surface. 2D-distribution of the mass flows in the grid of SDTrimSP-2D simulation after a projectile/recoil atom has slowed down.

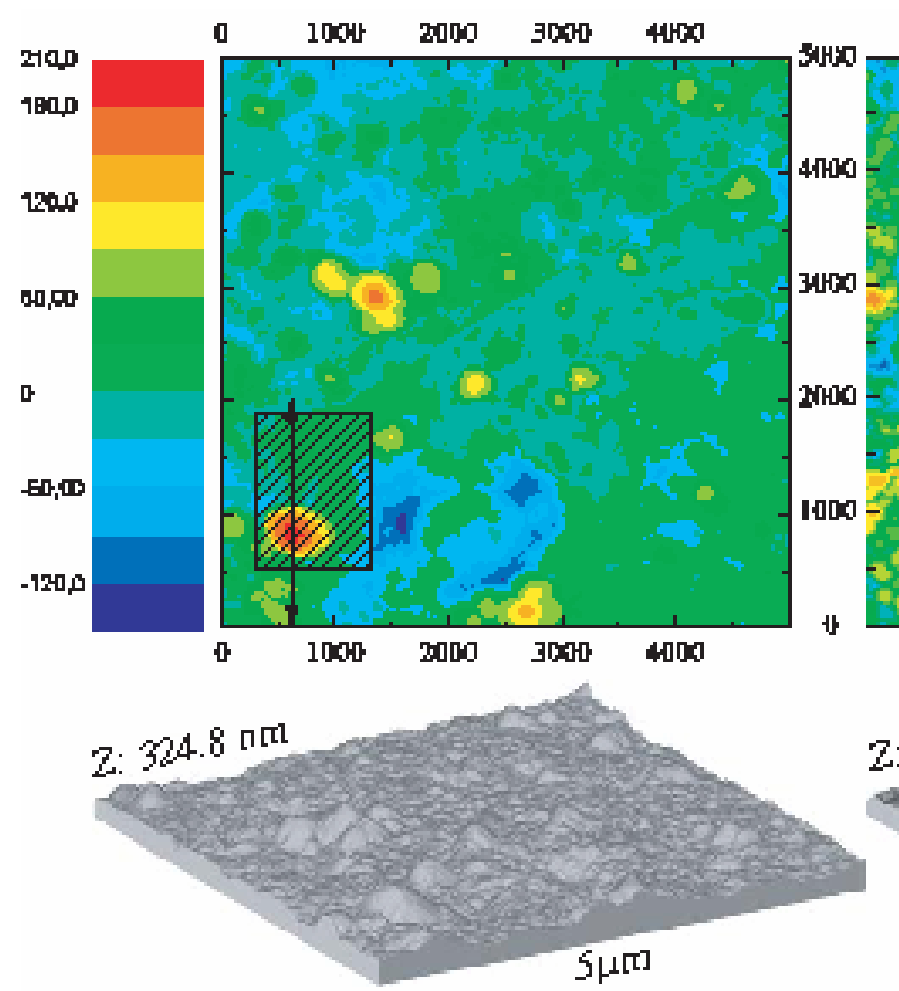

(a)
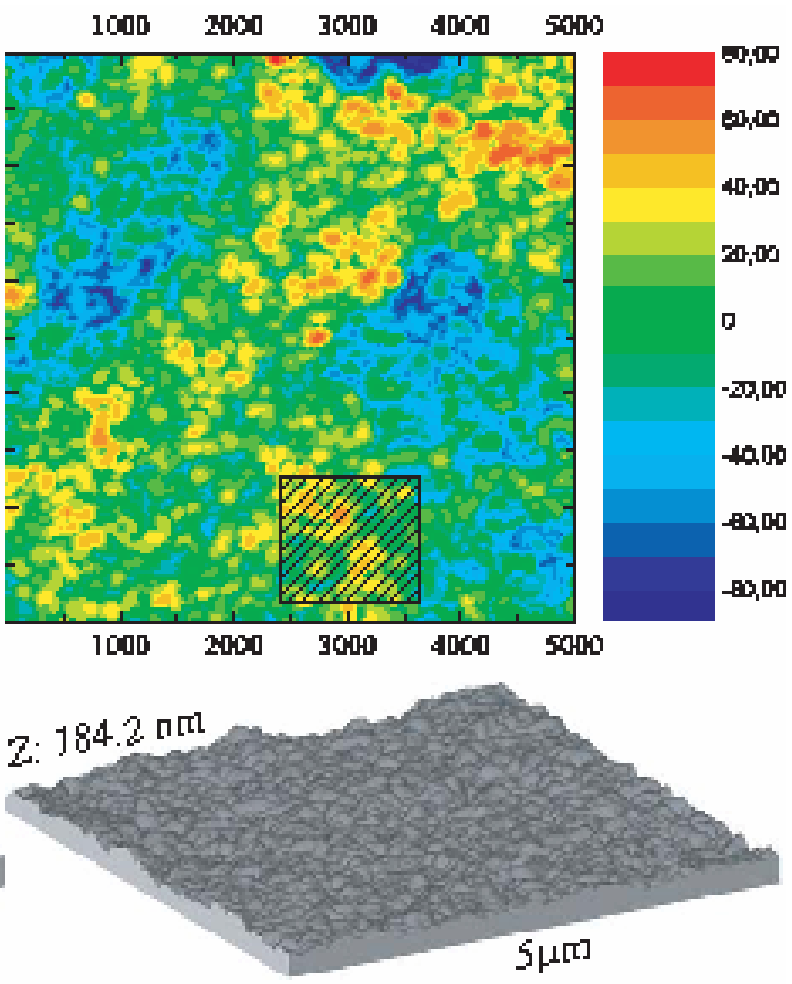

(b)

Figure 2. AFM images of the W layer sample surface. (a) - surface before bombardment; the arrow denotes the position of the surface 2-D cut used for the modelling; the hatched rectangle shows the surface part, which has been used for 3-D cut for quantitative comparison with results of the simulation. (b) - surface after the bombardment; the hatched rectangle shows the surface part, which has been used for 3-D cut for quantitative comparison with results of the simulation. 
Page 11

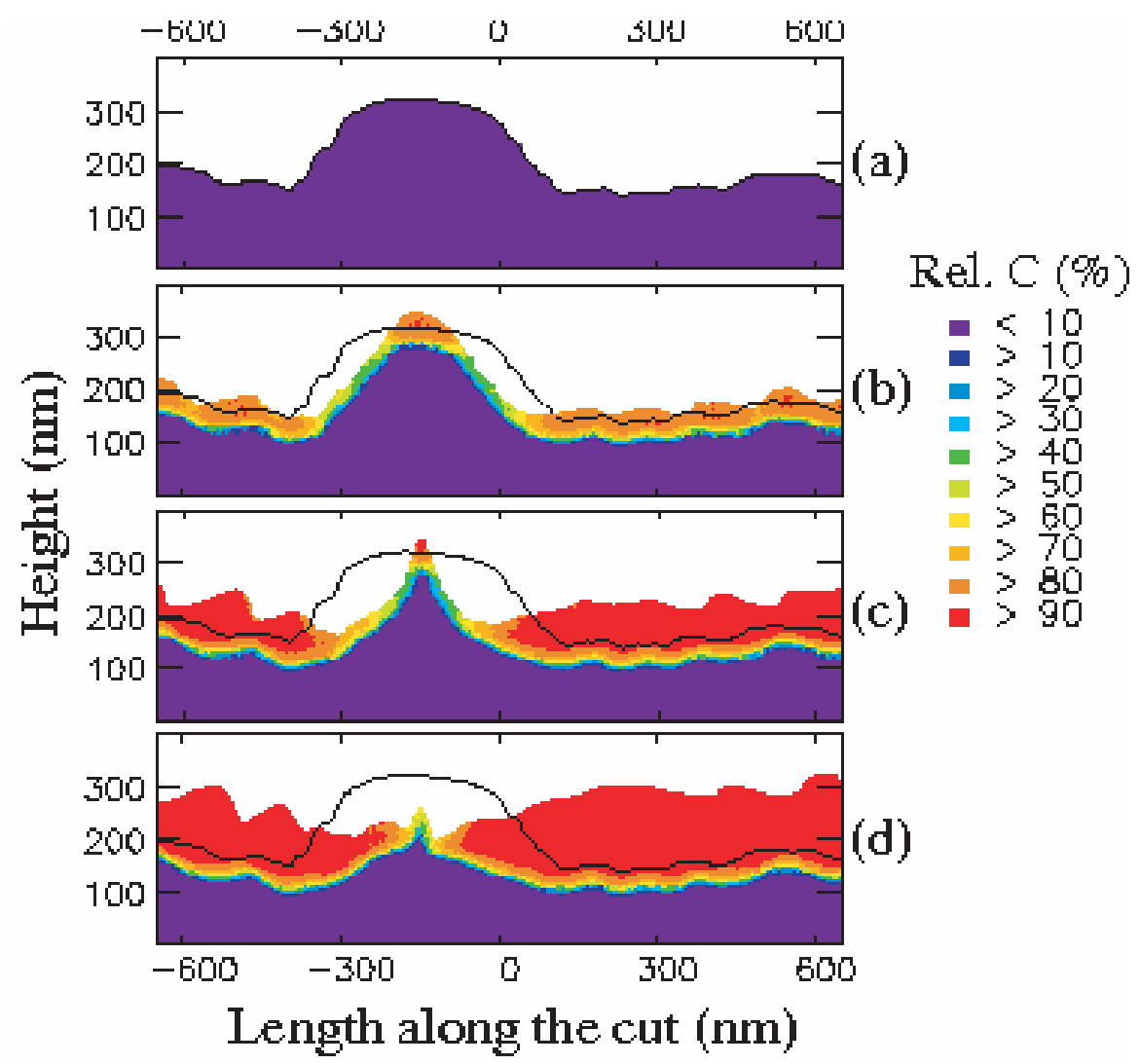

Figure 3. Numerical simulation of the evolution of the 2-D profile cut as a result of the ion bombardment. (a) - initial 2-D surface profile indicated in Figure 2 (a) by the arrow; (b) 2-D surface profile after bombardment with a C-fluence of $10^{18} \mathrm{~cm}^{-2}$; (c) - 2-D surface profile after a C-fluence of $2 \times 10^{18} \mathrm{~cm}^{-2} ;$ (d) -2 -D surface profile after the fluence of $3 \times 10^{18} \mathrm{~cm}^{-2}$.

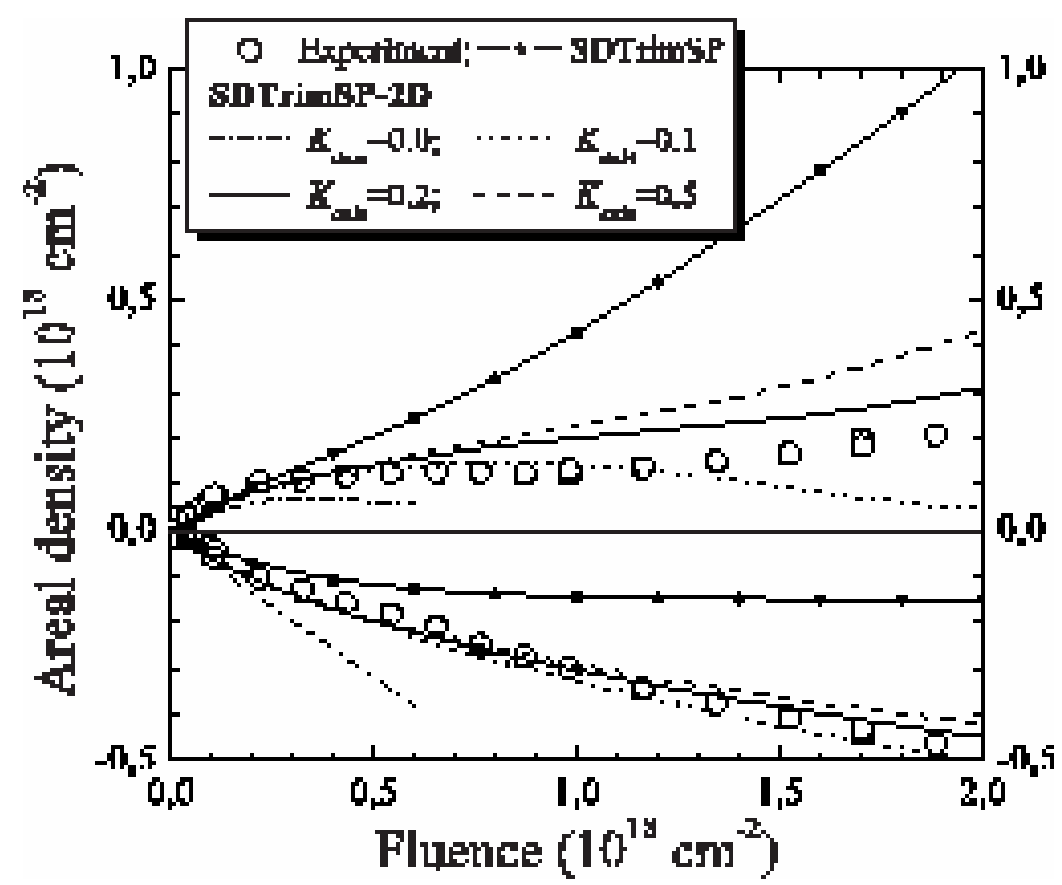

Figure 4. Evolution of elemental surface composition as a result of ion bombardment; the lines and data points with positive values correspond to the increase of the $\mathrm{C}$ areal density due to implantation, while the data with negative values correspond to the decrease of the $\mathrm{W}$ areal density due to sputtering. 
Page 12

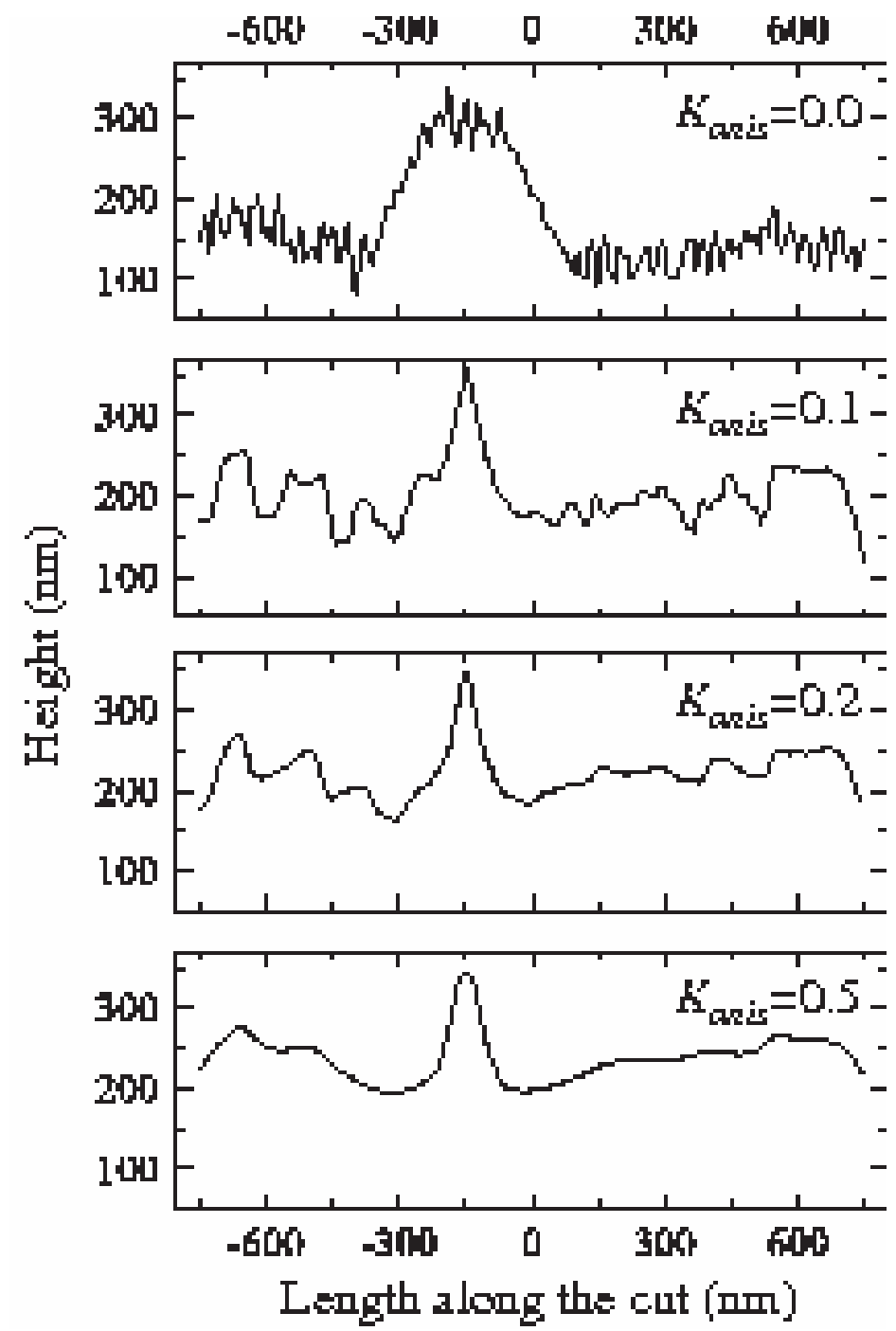

Figure 5. The influence of the anisotropy coefficient on the 2-D profile of the surface. (a) $=0.0 ;(\mathrm{b})-=0.1 ;(\mathrm{c})-=0.2 ;(\mathrm{d})-=0.5$. 


\section{Page 13}
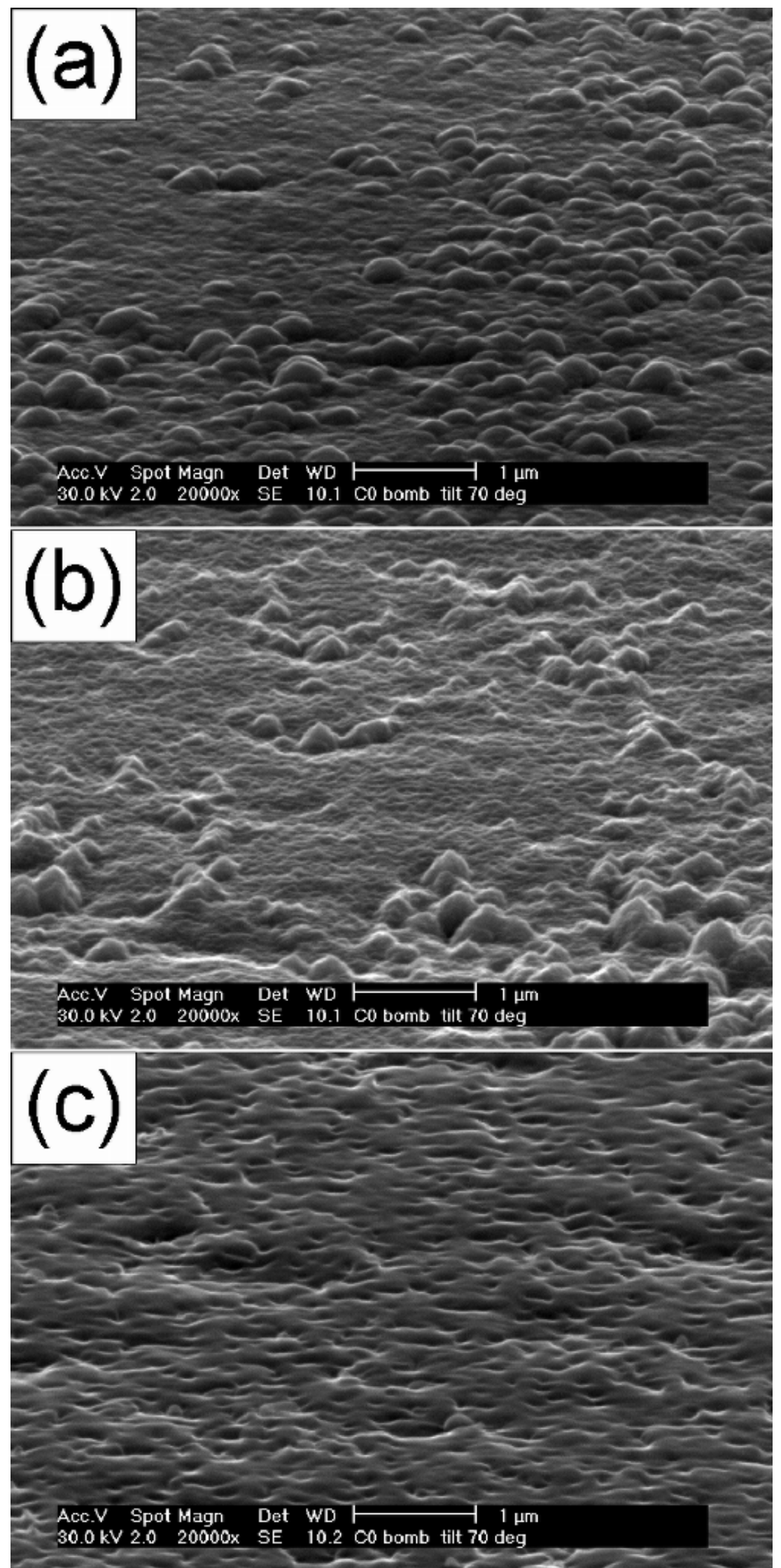

Figure 6. Evolution of the surface morphology observed at a tilt angle of $70^{\circ}$ by SEM. (a) initial state of the surface; (b) - surface after bombardment with an intermediate fluence; (c) - surface after almost complete coverage with ion beam deposited $\mathrm{C}$ layer. 


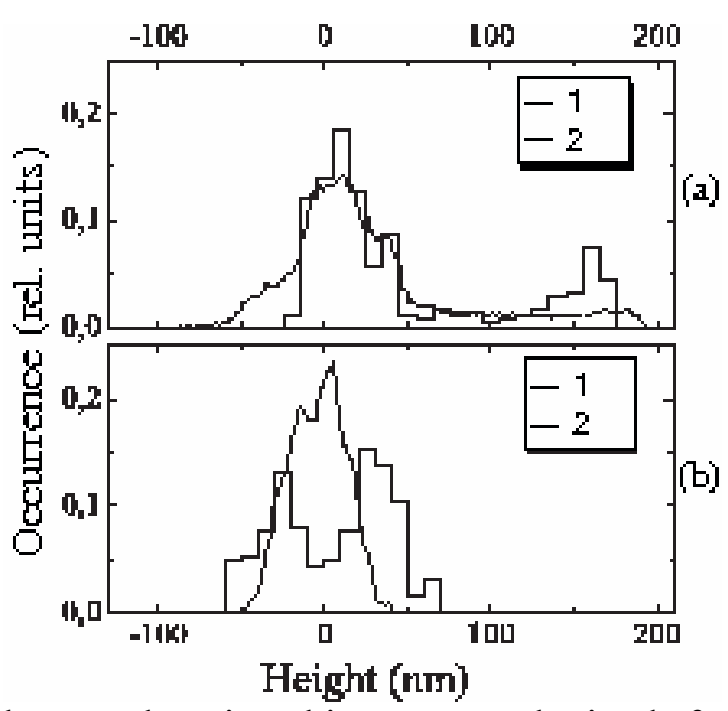

Figure 7. Surface roughness elevation histograms obtained from simulation and AFM measurements. (a): 1 - roughness before bombardment; experimental histogram calculated over the area marked by the rectangle in Figure 2(a), 2 - roughness before bombardment; experimental histogram calculated over the 2-D profile cut marked by the arrow in Figure 2(a). (b): 1 - roughness after bombardment; experimental histogram calculated over the area marked by the rectangle in Figure 2(b), 2 - histogram calculated over the profile plotted in Figure 3(d).

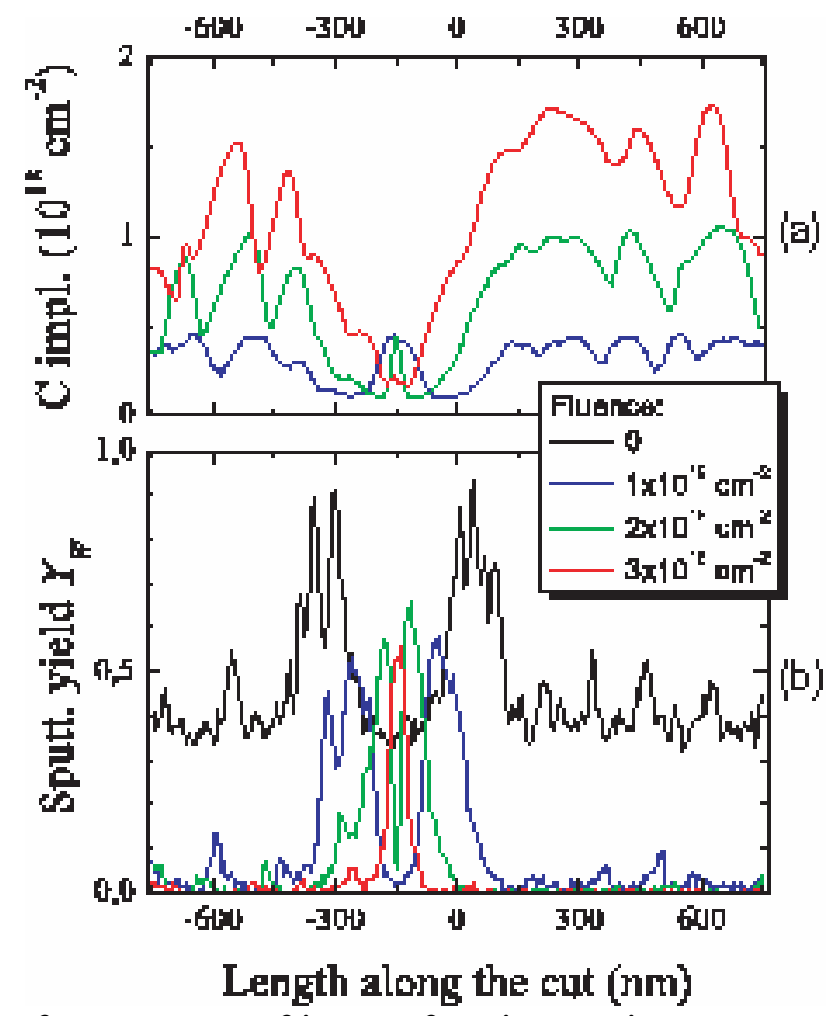

Figure 8. Dependency of parameters of ion-surface interactions on geometry. (a) - evolution of the local areal density of implanted $\mathrm{C}$ with fluence. (b) - evolution of the local sputtering yield of $\mathrm{W}$ with fluence. 